ÉGYPTE monde arabe

\section{Égypte/Monde arabe}

$15-16 \mid 1993$

Les crises soudanaises des années 80

\title{
Les mouvements de population provoqués par la sécheresse
}

Une approche microsociologique

\section{Ahmed Taha Mohammed}

\section{(2) OpenEdition}

\section{Journals}

Édition électronique

URL : https://journals.openedition.org/ema/1020

DOI : 10.4000/ema.1020

ISSN : 2090-7273

Éditeur

CEDEJ - Centre d'études et de documentation économiques juridiques et sociales

Édition imprimée

Date de publication : 31 décembre 1993

Pagination : 119-136

ISSN : 1110-5097

Référence électronique

Ahmed Taha Mohammed, "Les mouvements de population provoqués par la sécheresse », Égypte/ Monde arabe [En ligne], 15-16 | 1993, mis en ligne le 08 juillet 2008, consulté le 07 juillet 2022. URL :

http://journals.openedition.org/ema/1020 ; DOI : https://doi.org/10.4000/ema.1020

Ce document a été généré automatiquement le 7 juillet 2022.

Tous droits réservés 


\title{
Les mouvements de population provoqués par la sécheresse
}

\author{
Une approche microsociologique
}

\author{
Ahmed Taha Mohammed
}

\section{NOTE DE L'ÉDITEUR}

Traduit de l'anglais et adapté par François Ireton.

1 Le flux et le reflux de paysans et de travailleurs pauvres migrant sous la pression de conditions de vie désastreuses et dans l'espoir d'une vie meilleure, leur départdispersion et leur réinstallation-regroupement, constituent au Soudan un ensemble de phénomènes collectifs de première importance qui reflète l'ampleur des crises de tous ordres qu'a connues le pays ces dernières années (Mohammed, 1989). On distingue habituellement deux sortes de mouvements de population : ceux qui sont volontaires et ceux qui sont «involontaires" - c'est-à-dire résultant de rapports de forces défavorables aux migrants, qu'il s'agisse des exodes qui suivent catastrophes naturelles, défaites et invasions ou des déplacements entraînés par la traite de peuples vaincus ou capturés et réduits en esclavage. Ces migrations «involontaires» ont des effets multiples: ainsi, sous la période coloniale, un des facteurs de développement du marché du travail dans différents pays d'Afrique fut l'afflux d'une main-d'œuvre issue de déplacements et de regroupements forcés. Allan Booth (1982) a écrit: «L'histoire africaine est remplie d'exemples d'une force de travail recrutée sous la contrainte et entrant dans les rapports salariés aux conditions dictées par le Capital, avec le soutient de l'État ». Les migrations involontaires ne constituent donc pas un phénomène neuf en Afrique, et au Soudan en particulier.

2 La littérature portant sur ces questions fait actuellement preuve d'une nouvelle exigence dans la recherche de définitions prenant en compte la complexité des phénomènes de déplacements de population. Shami (1990), par exemple, tente de définir les déplacements "involontaires" de population comme "des processus 
collectifs de départ-réinstallation d'individus hors de leur habitat habituel, sous l'impact d'une force "supérieure ». Les trois agents majeurs de déplacement sont; l'action directe de l'État (lors de l'implantation de grands projets de développement par exemple), les guerres, soulèvements et persécutions, enfin les catastrophes naturelles. » Ces déplacements sont souvent, en fait, le produit d'une combinaison de facteurs, ceux qui viennent d'être énumérés étant les principaux. Durant ces dernières années, le Soudan les a tous trois expérimentés.

Dans cette étude, il sera question des déplacements dus à un type de "catastrophe naturelle » et, précisément, au départ-dispersion des populations de l'ouest du Soudan sous l'effet de la sécheresse. Bien que, dans ce cas, le facteur initial du départ soit un certain type de "force ", il n'en demeure pas moins que le déplacement relève d'une décision et que les unités sociales qui la prennent ont un certain degré et une certaine marge de « liberté ». Ainsi, dès qu'elle est appliquée à un cas concret - en l'occurrence, les déplacements effectués à la suite d'un certain type de catastrophe « naturelle »-, la dichotomie « volontaire/involontaire » semble peu opératoire. De plus, si l'on envisage les deux aspects, logiquement inséparables, de tout déplacement de population départ-dispersion et réinstallation-regroupement -, il semble que la composante «force» soit plus souvent (mais pas toujours) présente - ou présente avec plus d'intensité - dans le premier aspect que dans le second. La conceptualisation rigoureuse de ces aspects délicats des processus de déplacement n'a pas été suffisamment élaborée et varie selon les différentes doctrines sociologiques. Contentons-nous, pour montrer la variété des cas de figure sous ce rapport, de prendre quelques exemples empruntés à la réalité soudanaise :

- durant les années 60, les habitants de l'ancienne ville de Wadi Halfa ont dû la quitter suite à la construction du Haut-Barrage d'Assouan. Ils ont été réinstallés à l'est du Soudan, dans le périmètre agricole irrigué de New-Halfa qui leur était destiné. «Force » (physique: la montée des eaux) et/ou contrainte (économique et socio-politique) sont présentes, à des degrés divers, dans les processus de départ comme d'arrivée, mais certains habitants de l'ancienne ville ont néanmoins choisi de se réinstaller ailleurs ;

- dans le cas de la mise en place du périmètre irrigué agricole de Rahad, le départ était optionnel (les habitants pouvant rester dans leur village ou le quitter pour les terres nouvellement mises en culture), mais les lieux de réinstallation étaient strictement déterminés par les autorités ;

- la sécheresse a provoqué des départs « forcés » (mais certains ne sont pas partis), alors que le choix du lieu de réinstallation comportait pour les migrants une large « liberté ».

II va sans dire que l'intensité et l'impact de la «force » ou de la contrainte ne sont pas les mêmes (objectivement et subjectivement) selon les strates sociales, les types de groupes domestique et les lieux géographiques, à l'intérieur même d'une zone affectée de manière homogène par la sécheresse. Les marges de choix et degrés de liberté sont directement liés au niveau de développement atteint dans les différents lieux et aux caractéristiques des groupes concernés en matière de «capital» économique et humain. Par ailleurs, la saisie des variables permettant d'identifier ces différentiels de niveaux de développement et de caractéristiques familiales, dans les zones affectées par la sécheresse, rend possible la formulation et l'adoption de mesures visant, au plus bas coût politique et économique, des buts alternatifs déterminés (par exemple, maintien de la population sur le lieu d'arrivée ou encouragement au retour dans la zone d'origine, le tout dans les meilleures conditions). 
5 La politique actuellement suivie encourage le retour des familles victimes de la sécheresse vers leur village d'origine. L'on a donc choisi ici de comparer systématiquement, parmi les familles qui ont effectué un déplacement lors de la sécheresse, les caractéristiques socio-économiques d'un sous-échantillon de familles qui ont migré et résident toujours dans leur lieu d'arrivée (une ville, en l'occurrence), à celles d'un sous-échantillon de familles qui ont accompli, après un séjour dans la même ville, une migration-retour vers leur lieu d'origine. L'on testera donc, pour un ensemble de variables visant à décrire ces caractéristiques, l'existence de différences significatives entre ces deux sous-échantillons, de façon à pouvoir, par généralisation, identifier, au vu de leurs caractéristiques, les familles émigrées qui ont une bonne probabilité d'effectuer une migration-retour et celles qui resteront vraisemblablement sur place.

6 Cependant, une recherche sur les déplacements de population suite à la sécheresse qui ne tiendrait compte que des caractéristiques "post-sécheresse » des familles, sans s'interroger : (a) sur leur situation antérieure, (b) sur les relations entre cette situation et les manifestations socio-économiques de la sécheresse et (c) sur les modifications introduites dans cette situation par ces manifestations elles-mêmes et les déplacements qui en sont la conséquence, passerait à côté des objectifs tant scientifiques qu'appliqués » qu'elle s'est définis. Comme l'écrit Shami (op. cit.), plutôt qu'une réaction passive, les déplacements faisant suite à un désastre naturel ne doivent pas constituer de simples objets d'étude dont la connaissance permettrait de mieux organiser les secours. Ils sont révélateurs, dans leurs modalités et leur intensité, des conditions socio-économiques qui régnaient avant le désastre et qui déterminent en partie les conséquences de ce dernier. Toujours selon Shami (op. cit.), une telle étude ne doit pas préjuger de ce qui est cause ou conséquence de ces déplacements, mais prendre comme objet tout ce qui peut avoir l'un de ces deux statuts, et d'abord analyser la dynamique des forces sociales et de leurs aspirations, ainsi que celle des décisions politiques. Les déplacements de population et leurs conséquences sont en effet liés à des phénomènes qui relèvent de domaines où interviennent aussi bien les «développeurs" et les « modernisateurs » que les « opposants » et les « rebelles».

7 Dans cette étude, l'on se refusera donc à séparer les déplacements exceptionnels dus à la sécheresse de l'ensemble des autres déplacements pratiqués par la société étudiée, et d'abord des migrations pendulaires saisonnières qui mènent de nombreux habitants de l'ouest du Soudan vers les plantations cotonnières de la Guezira ou d'autres périmètres irrigués. Ce n'est qu'en mettant en relation ces deux types de déplacement qu'il sera possible d'isoler les causes propres au départ-dispersion, suite à la sécheresse, d'OuestSoudanais "réfugiés de l'environnement». Ces derniers, comme on tentera ici de le montrer, constituent en fait une « excroissance naturelle » de la migration saisonnière. De plus, en examinant $l^{\prime}$ » élasticité » de la réponse paysanne à la sécheresse, nous pourrons juger du degré d'efficacité ou d'inefficacité de la politique adoptée face à ces déplacements et contribuer à déterminer des mesures plus adaptées à ce qui peut être accepté en la matière par les agents sociaux concernés. Les principales questions auxquelles on tentera de répondre seront donc les suivantes:

- Quelles sont les différences significatives entre les caractéristiques socio-économiques des migrants du fait de la sécheresse qui sont retournés à leur lieu de résidence initiale, et les caractéristiques de ceux qui n'y sont pas retournés? 
- Quels sont les liens entre les départs-dispersions dus à l'irruption de la sécheresse et le processus de longue date et continu de migration saisonnière ? La réponse permettra de mesurer sous un angle nouveau les causes et conséquences profondes de la dégradation de l'environnement et de la réduction des possibilités de subsistance de la population de l'ouest du Soudan.

Contexte de l'étude

8 Au Soudan, l'espérance de vie à la naissance n'est que de 48 ans, et seulement $45 \%$ de la population adulte sait lire et écrire. L'on s'accorde généralement sur le fait que le niveau de vie moyen des habitants est en déclin depuis une quinzaine d'années et que l'inégalité de la distribution des revenus s'y est creusée, la pauvreté s'aggravant en termes d'écarts relatifs et s'élargissant en termes de prévalence. Le secteur de la production agricole prédomine, employant $80 \%$ de la population active et assurant 70 $\%$ de la valeur des revenus d'exportation. Le secteur agricole y est divisé en trois sousensembles: l'agriculture irriguée, l'agriculture pluviale mécanisée et l'agriculture pluviale traditionnelle. Les investissements publics ont favorisé le secteur non agricole par rapport au secteur agricole et, à l'intérieur de ce dernier, les agricultures irriguée et pluviale mécanisée par rapport à l'agriculture pluviale traditionnelle. La vague de sécheresse actuelle met en péril plus particulièrement ce dernier secteur qui n'a jamais fait l'objet, durant la période de pré-sécherese, d'aucune politique de gestion à long terme. Par ailleurs, on le sait, le Soudan fait de nouveau, depuis 1983, l'expérience d'une guerre civile qui, comme la sécheresse, provoque famine, misère et déplacements massifs de population.

C'est dès le début des années 60 que les spécialistes ont attiré l'attention sur les dangers de la désertification et sur l'arrivée probable de périodes de sécheresse et de famine dans la ceinture sub-saharienne de l'Afrique. Malgré des variations pluviométriques interannuelles d'amplitude accrue et un déclin du niveau moyen annuel des pluies depuis 1969, le Soudan n'a pas connu de situation dramatique sur les plans climatique et alimentaire durant les années 70. Ce n'est qu'au début des années 80 que la sécheresse a resserré nettement son étau, et les premiers déplacements de population lui étant directement liés ont fait leur apparition en 1983-84. Le déficit pluvial a varié selon les régions de la ceinture sahélienne du Soudan. Cependant, en 1984-85, il a été particulièrement important, et a été suivi d'un autre déficit en 1990-91. Selon une enquête entreprise en 1990 par le Ministère soudanais du Travail et non encore publiée, portant sur les migrations internes, près de $5 \%$ du total des migrants ( $9 \%$ de ceux qui résident à la campagne et $4 \%$ de ceux qui résident en ville) vers ou dans la partie nord du pays, désignent la sécheresse comme cause de leur migration. La région qui a été ,le plus fortement frappée par la sécheresse est l'ouest du Soudan, d'où vient la majorité des migrants saisonniers. Dans les années 60 et 70 cette région contribuait pour la moitié au PNB. Depuis le début des années 80 , elle est celle qui connaît les problèmes alimentaires les plus graves.

Parmi les lieux d'enquêtes possibles dans cette partie du Soudan, l'on a choisi al-Halba et sa périphérie immédiate. Cet ensemble est situé dans la province du Nil Blanc, à proximité de la limite est du Nord-Kordofan. Il s'agit d'une zone à la fois très affectée par la sécheresse et dont une grande partie de la population accomplit régulièrement des migrations saisonnières. La population qui en a émigré lors de la sécheresse s'est en général réinstallée à al-Dueim, capitale de la province du Nil Blanc.

Méthodologie et collecte des données 
11 Al-Halba et sa zone périphérique constituent une unité administrative de niveau inférieur (rural council) située à $70 \mathrm{~km}$ au sud-ouest d'al-Dueim. Al-Halba même compte 2.000 habitants et, dans un diamètre de $20 \mathrm{~km}$, on trouve quelques villages regroupant entre un peu moins de 100 à un peu moins de 1.000 habitants. Aucun de ces villages ne possède de point d'eau pérenne, d'unité de santé et de soins, d'école, de commerce fixe ni de bâtiment administratif. Tous ces villages dépendent, pour le service le plus élémentaire et la moindre marchandise, d'al-Halba ou d'al-Humora dans le NordKordofan. Les activités commerciales de la zone sont concentrées à al-Halba, où se tient un marché hebdomadaire vers lequel converge la population de la périphérie (le marché d'al-Humora se tenant un autre jour de la semaine). AI-Halba est située sur l'axe allant d'Omdurman (partie ouest de l'agglomération de Khartoum) à al-Obeid (capitale du Kordofan). Entre 20 et 30 camions et bus passent journellement par alHalba, venant de ou allant à Omdurman. Ce mouvement représente une des sources principales de revenus des familles d'al-Halba, les femmes (parfois les hommes) tenant quelques buvettes et restaurants.

12 La zone d'al-Halba se caractérise par un sol sableux parsemé de dunes. Le sorgho, le millet et le sésame, principales cultures, sont produits dans de petites exploitations. Au moment de l'enquête (fin de la saison des pluies, soit septembre 1990), il était clair que la récolte serait mauvaise et que l'on allait vers un déficit alimentaire dû essentiellement à une trop faible pluviométrie durant l'été. On pouvait s'attendre à ce qu'une partie de la population des villages périphériques d'al-Halba quitte la région si la sécheresse durait.

13 Dans la zone retenue pour l'enquête, c'est le village de Lgaïdat al-Taïr qui a été choisi, les familles qui le composent ayant effectué une migration de longue durée (c'est-à-dire non saisonnière) et beaucoup étant revenues à leur lieu d'origine. Situé à $12 \mathrm{~km}$ à l'est d'al-Halba, ce village est peuplé de 67 familles qui, démunies d'eau potable, doivent aller la chercher à une distance nécessitant un trajet de 7 heures à dos d'âne. Cette population correspond à la définition d'un des groupes visés par l'enquête : la quasitotalité des habitants a quitté son lieu de résidence durant les années de sécheresse de 1983-84 et 1984-85 et a migré vers al-Dueim. Beaucoup sont revenus habiter le village quelques années après, mais une proportion non négligeable de ces migrants sont restés à al-Dueim, où ils résident encore actuellement. Sur les 67 familles que compte actuellement le village, on a tiré un échantillon aléatoire de 28 familles et interrogé leurs chefs de ménage (en général des hommes).

14 A al-Dueim, 72 familles toutes originaires de la zone d'al-Halba (dont celles venues de Lgaïdat al-Taïr) ont été enquêtées. L'enquête s'adressait donc à deux sous-ensembles : 28 familles ayant accompli une migration-retour à partir d'al-Dueim vers leur village d'origine (toutes originaires du village de Lgaïdat al-Tair) et 72 familles originaires de la zone d'al-Halba (dont un certain nombre de Lgaïdat al-Taïr) et résidant à al-Dueim. Il était ainsi aisé de définir statistiquement les caractéristiques socio-économiques différenciant les familles revenues à leur point de départ et celles demeurées dans la capitale de la province. De plus, une partie des hommes appartenant aux familles immigrées à al-Dueim ont des emplois saisonniers hors de cette ville pour compléter les revenus de leur travail en zone urbaine. Le maintien à al-Dueim peut ainsi être lié à ces migrations saisonnières, hypothèse que l'enquête a également tenté de vérifier.

Les cent familles enquêtées rassemblent un total de 678 individus dont 325 femmes. On y compte 352 adultes de plus de 15 ans- Près de $90 \%$ des familles retournées au village 
avaient migré à al-Dueim lors des années de sécheresse intense (1984 et 85) et $75 \%$ d'entre elles sont restées en ville entre un et cinq ans ( $25 \%$ moins d'un an). Un peu moins des deux tiers des familles restées en ville sont arrivées lors de ces années, mais $92 \%$ résident à al-Dueim depuis cinq ans et plus, ce qui veut dire que près d'un tiers a migré avant la vague de sécheresse. La proportion de familles ayant quitté leur village alors que la sécheresse y sévissait ou y avait sévi - ce qui semble indiquer une migration sous la pression des conséquences économiques de la sécheresse - est donc nettement plus élevée chez les migrants-retour (elle confine aux $100 \%$ ) que chez ceux qui sont restés en ville (un peu plus de $70 \%$ ). L'émigration post-sécheresse ou simultanée à celle-ci, migration dite "sous contrainte", a ainsi été suivie soit d'un retour dès que cette contrainte fut considérée comme relâchée, soit d'un maintien en ville, alors que la migration pré-sécheresse peut être assimilée à un acte "volontaire " d'immigration en ville, dont on suppose qu'elle a beaucoup plus de chance d'être suivie d'un établissement définitif. Toutes les variables caractérisant les familles sont croisées avec les deux modalités de leur trajectoire migratoire (présence/absence d'une migration-retour).

Activités principales des deux groupes de migrants

\begin{tabular}{|c|c|c|c|c|c|c|c|c|c|c|c|}
\hline $\begin{array}{l}\text { Groupes } \\
\text { concernés }\end{array}$ & Chômage & $\begin{array}{l}\text { Agriculture } \\
\text { Elevage }\end{array}$ & \multicolumn{3}{|c|}{$\begin{array}{l}\text { Travail } \\
\text { salarié } \\
\text { irrégulier }\end{array}$} & \multicolumn{3}{|c|}{$\begin{array}{l}\text { Travail } \\
\text { salarié } \\
\text { régulie }\end{array}$} & $\begin{array}{l}\text { Commerce } \\
\text { Artisanat }\end{array}$ & Autres & Total \\
\hline \multirow{2}{*}{$\begin{array}{l}\text { Avant } \\
\text { sécheresse }\end{array}$} & \multicolumn{2}{|c|}{ Migrants retour } & - & $100 \%$ & 0 & 0 & 0 & 0 & $100 \%$ & & \\
\hline & \multicolumn{2}{|c|}{ Migrants installés en ville } & $1 \%$ & $59 \%$ & $11 \%$ & $1 \%$ & $\% 24 \%$ & $4 \%$ & $100 \%$ & & \\
\hline \multirow{2}{*}{$\begin{array}{l}\text { Après } \\
\text { sécheresse }\end{array}$} & \multicolumn{2}{|c|}{ Migrants retour } & \multicolumn{2}{|c|}{$-96 \%$} & $4 \%$ & 0 & 0 & 0 & $100 \%$ & & \\
\hline & \multicolumn{2}{|c|}{ Migrants installés en ville } & -1 & $10 \%$ & $33 \%$ & $1 \%$ & $35 \%$ & $21 \%$ & $100 \%$ & & \\
\hline \multirow{2}{*}{$\begin{array}{l}\text { Semaine } \\
\text { précédant la } \\
\text { sécheresse }\end{array}$} & \multicolumn{2}{|c|}{. Migrants retour } & $50 \%$ & $4 \%$ & $21 \%$ & 0 & $14 \%$ & $11 \%$ & $100 \%$ & & \\
\hline & \multicolumn{2}{|c|}{ Migrants installés en ville } & $11 \%$ & $3 \%$ & $32 \%$ & $1 \%$ & $32 \%$ & $21 \%$ & $100 \%$ & & \\
\hline
\end{tabular}

Les variables socio-démographiques

Celles-ci concernent la taille du ménage, le nombre d'individus de genre féminin et le nombre de personnes de 15 ans et moins entrant dans sa composition (indicateur, par approximation, du taux de dépendance, puisque le nombre d'adultes de plus de 65 ans est quasi-négligeable), l'âge du chef de ménage, la mortalité infantile (des enfants de 5 ans et moins) parmi les enfants nés vivants des femmes de l'échantillon mariées au moment de l'enquête, la structure (nucléaire/élargie) de la famille et l'existence ou non d'un divorce survenu dans la famille depuis sa migration hors de la zone d'al-Halba. Aucun croisement de ces variables avec les deux modalités du statut migratoire ne fournit, lors de l'application du test de « Khi carré », de différence significative au seuil 
de $5 \%$ entre migrants-retour et migrants simples. Trois croisements en donnent une, faible, au seuil de $10 \%$ : la taille du ménage, la mortalité infantile et l'existence d'un divorce dans l'histoire de la famille.

Plus la taille du ménage excède la taille moyenne des familles de l'échantillon $(6,8)$ et plus les familles ont de chance d'avoir accompli une migration-retour, ce que l'on peut interpréter comme la conséquence de la difficulté des familles nombreuses à s'adapter au mode de vie urbain.

Plus la famille a connu une mortalité infantile importante et plus elle a également de chance d'être retournée dans son village d'origine.

Les études sur les migrations faisant suite à des catastrophes "naturelles ", dont la sécheresse et la famine, mettent souvent en relief le fait que le taux d'éclatement des familles est plus élevé chez celles qui ont accompli une migration définitive que chez celles qui, passée la période la plus tragique, regagnent au plus vite leur lieu d'origine. Ici, le divorce (intervenu dans les, sept années précédant l'enquête) a été retenu comme indicateur d'éclatement de la famille. Parmi les ménages qui ont effectué une migration-retour, seulement $7 \%$ ont connu un divorce depuis 1983, contre $22 \%$ parmi ceux restés à al-Dueim $\left(\mathrm{Khi}^{2}\right.$ : diff.sign.; seuil : 10\%).

Les variables socio-économiques

20 Ces variables ont été divisées en trois sous-ensembles : le type d'activité professionnelle (simplifiée), le patrimoine, les modes et niveaux de consommation.

Occupation et catégorie professionnelle

21 L'on a pris en compte ici les occupations " professionnelles " principales juste avant la migration, juste après, et durant la semaine précédant l'enquête (cf. tableau ci-dessus).

$100 \%$ des familles retournées dans leur village d'origine pratiquaient l'agriculture et/ ou l'élevage avant leur première migration, alors que ce n'était » le cas que de $60 \%$ des familles ayant accompli une migration simple ( $24 \%$ de ces dernières étaient, au village, engagées dans des activités commerciales ou artisanales et $12 \%$ des activités salariées irrégulières). L'on peut penser que le fait de pratiquer des activités agricoles a poussé une forte proportion d'émigrés à retourner dans leur village d'origine, où ils possèdent des terres, alors que les activités salariées irrégulières, le commerce et l'artisanal sont non seulement moins ou peu liés à un patrimoine fixe, mais supposent souvent l'existence de réseaux de relations qui ont pu faciliter l'installation et le maintien en ville ( $\mathrm{Khi}^{2}$ : diff. sign. ; seuil : $\left.2 \%\right)$.

En ce qui concerne le type de profession principale exercée par le chef de famille après la migration, $96 \%$ des familles revenues au village ont retrouvé leur activité agricole, alors que les familles restées à al-Dueim ne sont plus que $9 \%$ à la pratiquer (contre $60 \%$ avant leur migration). Parmi les familles ayant immigré et résidant dans cette ville, par contre, les emplois salariés irréguliers sont passés de $12 \%$ avant la migration à $32 \%$ après cette dernière, tandis que la représentation des activités commerciales et artisanales croît également, passant de $24 \%$ à $35 \%$, les « autres emplois » connaissant la même évolution (de $4 \%$ à $21 \%$ ). L'installation « définitive » en ville correspond donc à une forte mobilité professionnelle; s'agit-il - et dans quelles proportions - de mobilité ascendante ou descendante ? La réponse à cette question supposerait d'autres informations (revenus avant et après la migration, etc.) $\left(\mathrm{Khi}^{2}\right.$ : diff. sign.; seuil : $\left.1 \%\right)$.

Enfin, l'occupation exercée dans la semaine précédant l'enquête donne des résultats bien différents de ceux de la dernière variable examinée, pour les migrants retournés 
au village du moins; parmi ces derniers en effet, $50 \%$ des chefs de ménage n'ont exercé, durant ce laps de temps, aucune activité rapportant un revenu, contre seulement $11 \%$ parmi ceux qui se sont installés à al-Dueim. Ceci reflète à l'évidence les différences de volume et de structure de l'offre d'emploi en ville et à la campagne, pratiquement aucune autre offre que celle d'un travail agricole n'existant dans cette dernière - et encore ne s'agit-il que d'offre saisonnière. Ainsi, pour esquisser une réponse à la question posée plus haut, le passage d'une activité agricole n'occupant le paysan qu'une faible partie de l'année dans son village d'origine, à une succession d'emplois salariés irréguliers dont l'offre est, en ville, importante, ne signifie-t-il pas une mobilité professionnelle descendante ? $32 \%$ des migrants restés en ville déclarent avoir effectué des tâches ponctuelles contre rémunération durant la semaine précédant l'enquête; la même proportion dit avoir exercé des activités commerciales, et $21 \%$ évoquent des activités professionnelles "autres »; les $50 \%$ de migrants-retour ayant mené au village, durant cette même semaine, une activité économique se partagent entre les activités salariées irrégulières: 21 \% (très probablement agricoles), le commerce et l'artisanat $(14 \%)$ et les activités « autres» $(11 \%) ;\left(\mathrm{Khi}^{2}\right.$ : diff. sign. ; seuil : $1 \%)$.

Le contraste est évidemment frappant entre la stabilité qui prévaut, de l'immédiate après-migration jusqu'au moment de l'enquête, dans la répartition des types d'activité professionnelle des migrants installés en ville et les variations connues, dans ce domaine et pour la même période, par les migrants retournés au village. La stabilité et la continuité, si ce n'est dans un emploi donné, du moins dans l'exercice d'une activité professionnelle d'un certain type, distinguent les conditions d'existence en ville des migrants qui y sont restés, des conditions précaires d'emploi des migrants retournés au village et dont l'activité principale ne suffit à assurer ni la subsistance de la famille ni un emploi "à plein temps", même si elle est agricole et s'exerce sur leur propre exploitation avec leurs propres moyens de production. Ce sont les variables concernant ces derniers, et qui constituent le second sous-ensemble de variables socioéconomiques, dont on va maintenant examiner le croisement avec le statut migratoire. Patrimoine et moyens de production

26 Ces variables concernent la propriété d'une maison sur le lieu de résidence, la possession de terres agricoles et celle de bétail (ovins, caprins, ânes, chameaux) considérée comme une forme d'accumulation économique.

27 La quasi-totalité des migrants retournés au village y sont propriétaires d'une maison (96\%). L'enquête n'a pas permis de connaître la proportion d'immigrés à al-Dueim qui possédaient (ou possèdent encore) une maison dans leur village d'origine. Ce que l'on sait, c'est qu'ils ne sont que $26 \%$ a être propriétaires d'une maison dans cette ville; il s'agit d'immigrés qui avaient pour la plupart déjà tissé un réseau de relations à alDueim et qui s'y sont établis depuis longtemps. Les autres, soit presque les trois-quarts, sont locataires et payent entre 70 et 300 livres soudanaises (LS, fin 1990) par mois (157 LS en moyenne); il s'agit de maisons de brique crue, construites en zone réglementée (seulement $1 \%$ de maisons sont construites en zone "informelle»), mais en général démunies d'eau courante, d'électricité et le plus souvent de latrines privées $\left(\mathrm{Khi}^{2}\right.$ : diff. sign. ; seuil : $1 \%$ ).

28 S'agissant des terres agricoles possédées, elles sont toujours situées au village d'origine. Seulement $4 \%$ des migrants-retour en sont démunis, contre $15 \%$ des immigrés en ville, (ce qui reste faible). Ceux qui possèdent une superficie comprise entre 1 et 20 feddans 
représentent $15 \%$ des deux catégories de migrants. Une nette opposition se dessine cependant entre ces dernières en ce qui concerne la possession de plus de 20 feddans : $21 \%$ de migrants-retour jouissent de 20 à 40 feddans, contre $40 \%$ d'immigrés urbains, et $61 \%$ des premiers ont plus de 40 feddans contre seulement $30 \%$ des seconds. Il semble clair, ici, que la possession d'une superficie importante de terres (plus de 40 feddans) constitue un net encouragement au retour au village, une exploitation de cette taille permettant, avec des conditions climatiques "normales", d'assurer aisément la subsistance et l'emploi des membres du groupe domestique $\left(\mathrm{Khi}^{2}\right.$ : diff. sign. ; seuil : $5 \%$ ).

Le bétail, dans le cadre de l'économie agro-pastorale sahélienne « traditionnelle », est à la fois source et accumulation de richesses et moyen de production. Il était donc important d'intégrer dans cette enquête des variables le concernant. Vu les années de sécheresse qui, à partir de 1983, ont décimé les troupeaux et vu la lenteur de reconstitution de ces derniers, les variables utilisées sont simplement dichotomiques : on a retenu le fait que des membres des deux catégories de migrants possèdent ou ne possèdent pas d'ovins et de caprins, d'ânes, et de chameaux. $75 \%$ des ruraux d'origine revenus au village possèdent ovins et caprins, mais - contrairement à ce à quoi l'on pourrait s'attendre - $36 \%$ des immigrés urbains en ont également gardé (il s'agit en général de petits troupeaux qu'un membre de la famille peut faire pâturer à proximité de la ville, ou qui sont confiés à un berger ayant en charge des troupeaux appartenant à plusieurs propriétaires) ; $72 \%$ des premiers ont également au moins un âne, animal de bât par excellence, contre seulement 17 \% des seconds, alors que, vu ses fonctions, cet animal garde toute son utilité en ville. Il faut probablement y voir la conséquence de difficultés à le nourrir, la cherté du fourrage, etc. (Ces deux variables introduisent une différence significative entre les deux groupes de migrants, estimée par le test du Khi carré, au seuil de $1 \%$ ). Quant aux chameaux, dont la propriété constitue une véritable sécurité financière (capital sur pied important) et une source de rapport, les immigrés en ville n'en possèdent pas et les migrants-retour ne sont que $11 \%$ à en être propriétaires ( $\mathrm{Khi}^{2}$ : diff. sign. ; seuil : $5 \%$ ). Les pertes de bétail entre l'avant et l'aprèsmigration concernent presque autant les premiers $(79 \%)$ que les seconds $(68 \%)$ : pas de différence significative.

Si des corrélations apparaissent bien entre statut migratoire et possession de différents éléments de patrimoine agricole, les liens de causalité entre ces deux ordres de variables sont difficiles à établir de façon univoque, mis à part la liaison entre taille de l'exploitation agricole située au village et propension au retour. En l'absence, dans le questionnaire d'enquête, d'une question portant sur la possession, par les migrants installés en ville, d'une maison dans leur village d'origine, il n'est pas possible de tester le poids de cette variable en tant que facteur poussant au retour. Quant à la possession ou non de bétail, il est difficile de savoir - compte tenu du fait que le taux de migrants ayant subi des pertes dans ce domaine est à peu près constant quel que soit leur statut migratoire - si les migrants installés en ville y sont restés parce qu'ils n'avaient pas ou plus de bétail ou si, ayant décidé de rester en ville, il s'en sont dessaisis, en en réalisant par exemple la valeur dans l'achat d'autres biens. La possession d'un patrimoine autre que foncier (bien de production et/ou "richesse» accumulée) restant un facteur ambigu quant à son impact sur la probabilité d'accomplir une migration-retour au village ou de s'installer en ville, il peut être intéressant de croiser avec ces mêmes 
modalités de statut migratoire des variables renvoyant au mode et niveau de consommation que l'on peut considérer comme des indicateurs de pauvreté relative.

Modes et niveaux de consommation

31 Comme Kakwani (1990) et Galawee (1990) l'ont fait remarquer dans leurs travaux, effectués pour la Banque mondiale dans le cadre d'études sur l'évaluation des standards de vie (Living Standards Measurement Studies, LSMS), la mesure de la pauvreté relative peut être menée à partir du volume et de la structure des consommations domestiques, en particulier dans le cas de ménages vivant en-dessous d'un seuil de pauvreté déterminé. L'on tiendra compte de l'existence de biens durables et non durables. La population enquêtée ayant été victime des années de sécheresse et la plus grande partie d'entre elle ayant un niveau de vie situé à proximité ou en-dessous du seuil de pauvreté, les deux variables retenues pour appréhender la consommation de biens durables concernent l'achat de vêtements durant l'année qui a précédé l'enquête et d'ustensiles de cuisine durant les trois années antérieures (variables dichotomique: achat/non achat).

$75 \%$ des migrants retournés au village n'ont pas acheté de vêtements et $93 \%$ n'ont pas acquis d'ustensiles de cuisine durant ces périodes, contre respectivement $43 \%$ et $58 \%$ de migrants restés en ville ( $\mathrm{Khi}^{2}$ : diff. sign.; seuil : $1 \%$ ). Il semble clair, au vu de ces résultats, que - s'agissant ici d'achats de biens durables dits " de première nécessité " - le niveau d'équipement domestique des migrants urbains est moins sommaire que celui des migrants-retour, ce qui n'est pas nécessairement indicatif d'un meilleur niveau de vie global, mais de moins faibles disponibilités en numéraire (notons que l'auto-production domestique de vêtements et, à plus forte raison, d'ustensiles de cuisine par les ruraux est très rare).

33 S'agissant de consommation de biens non durables, les indicateurs suivants ont été choisis : la valeur monétaire des denrées alimentaires achetées la veille de l'enquête et la disponibilité ou non, au moment de l'enquête, d'une provision de céréales (sorgho et millet) susceptible d'assurer la subsistance de la famille pour une semaine. Alors que 60 $\%$ des familles de migrants urbains ont acheté la veille pour plus de 40 LS de céréales (lors de l'enquête, la quantité de farine de sorgho nécessaire à la subsistance d'une famille de taille moyenne - soit 6,9 membres dans notre échantillon - était de $25 \mathrm{LS}$ ), seules $14 \%$ des familles retournées au village avaient acquis des céréales pour la même somme ; néanmoins, $43 \%$ de ces familles avaient des réserves de grain pour au moins une semaine, alors que ce n'était le cas que de $15 \%$ des familles de migrants urbains (pour ces deux variables, $\mathrm{Khi}^{2}$; diff. sign. ; seuil : $1 \%$ ).

L'analyse sommaire de la consommation et des réserves de céréales ne peut, en ellemême, permettre de différencier des niveaux de consommation, mais bien plutôt des modes d'acquisition des denrées consommées, renvoyant - ce à quoi l'on pouvait s'attendre - à l'insertion dans des contextes économiques différents; les migrantsretour ruraux sont pratiquement tous engagés dans des activités agricoles, possèdent davantage de réserves de grain et en achètent beaucoup moins quotidiennement (notons que l'enquête a été effectuée avant la moisson, donc en pleine période de soudure, ce qui peut expliquer qu'une forte proportion de familles rurales (58 \%) n'aient pas de réserves céréalières pour une semaine; une partie de ces familles sont engagées dans des activités salariées agricoles et $40 \%$ des familles rurales ont acheté la veille pour 20 à 40 LS de grains, ce qui peut leur permettre de subsister). Les urbains, eux, dont beaucoup sont salariés - même s'il s'agit d'emplois précaires - ou disposent 
de revenus monétaires - venus du secteur informel, le plus souvent - n'ont que fort peu de réserves, mais ont un numéraire leur permettant des achats quotidiens de céréales en quantité suffisante.

En termes de niveau de consommation alimentaire (indicatrice de celle de biens non durables), les différences entre sous-catégories à l'intérieur de chacun des deux groupes de migrants sont sans doute supérieures à celles qui existent entre ces deux derniers considérés globalement; par contre, les consommations de biens durables donnent l'avantage aux migrants urbains. Si l'on joint à cet avantage celui que procure l'accès moins difficile à des emplois salariés - mêmes instables - ou métiers "indépendants" assurant tout au long de l'année des disponibilités financières minimales, il est possible d'affirmer que le niveau de vie global et les conditions d'existence sont un peu moins mauvaises pour les migrants urbains que pour ceux qui sont retournés au village.

Les variables socioculturelles

36 L'alphabétisation des membres de la familles (ou de certains d'entre eux) et la scolarisation des enfants sont des caractéristiques qui peuvent contribuer à expliquer le maintien en ville. Le fait de savoir lire et écrire peut favoriser l'accès à certains emplois et une meilleure adaptation à la vie urbaine ; la scolarisation des enfants, que permet la résidence en ville (il n'y a d'écoles, dans la zone d'origine, qu'à al-Halba), peut être un facteur encourageant la décision de s'y maintenir, ceci pour une double raison : ne pas « déscolariser » les enfants et miser sur leur avenir en ville en tant que diplômés - même à un niveau modeste. L'on constate que la quasi-totalité des membres (de plus de 10 ans) des familles retournées au village sont illettrés ; $4 \%$ des familles seulement ont un membre sachant lire ; $24 \%$ des familles migrantes installées en ville ont un ou deux membres alphabétisés ( $\mathrm{Khi}^{2}$ : diff. sign. ; seuil : $\left.10 \%\right)$. Seulement $7 \%$ des premières ont un enfant scolarisé, alors que c'est le cas pour $39 \%$ des secondes (Khi ${ }^{2}$ : diff. sign.; seuil : $5 \%$ ). Le contraste entre les deux groupes pris globalement est ici assez marqué. En termes de causalité, le fait d'être alphabétisé constitue bien un facteur incitant à rester en ville ; par contre, il aurait fallu connaître la proportion de familles retournées au village ayant eu un enfant scolarisé en ville avant d'en repartir, pour pouvoir tester dans quelle mesure la scolarisation constitue également un facteur favorisant l'installation en ville (les familles retournées au village peuvent en effet n'avoir jamais scolarisé leurs enfants - par absence de préoccupation dans ce domaine ou dans la perspective de la ré émigration - ou peuvent l'avoir fait mais avoir préféré ré émigrer, quitte à sacrifier la scolarisation; dans ce dernier cas, celle-ci ne constituerait pas par elle-même un facteur de maintien en ville). Par ailleurs, le niveau moins faible d'alphabétisation des migrants restés en ville est à mettre en relation avec leur accès plus important aux emplois qualifiés, après immigration.

Les facteurs de la migration

Il est commode de distinguer les causes directes de la migration, que seraient les décisions prises par des individus ou des ménages dans un contexte mental et matériel donné, et les causes indirectes et structurelles qui résideraient précisément dans ces contextes, essentiellement socio-économiques et sociopolitiques. Shami (op. cit.) déclare qu' « une migration doit être envisagée comme le point culminant d'un processus animé par des forces activées bien avant $e$ déplacement physique lui-même. »

L'on a choisi d'interroger les familles enquêtées sur ce qu'elles perçoivent comme étant la cause directe la plus importante de leur déplacement : la sécheresse en elle-même 
(pas - ou très peu - de récolte ou de pâturages), ou le manque de sources de revenu et/ou d'opportunité d'emploi (qui peuvent être, pour une part, des conséquences directes de la sécheresse), ou l'absence d'eau potable dans un périmètre considéré comme "raisonnable» (plus une rubrique « autres»). Il est intéressant de constater que les migrants retournés au village, dont on a vu qu'ils étaient tous engagés dans des activités agricoles avant leur première migration, sont nettement plus nombreux (57 \%) à donner comme cause directe de leur départ la sécheresse elle-même (ce qui concorde également avec le fait, examiné plus haut, que toutes les familles appartenant à cette catégorie de migrants n'ont quitté leur village qu'au moment de - ou après - l'année de grande sécheresse). Les migrants restés en ville ne sont que $31 \%$ à donner la sécheresse comme cause première de leur migration. La faiblesse des revenus et des opportunités d'emploi est à peu près également alléguée par les deux groupes (autour de $20 \%$ ) ; par contre, l'absence d'eau potable au village est plus souvent évoquée par les migrants urbains ( $25 \%$ - ils répondent dans la même proportion par " autres causes ») que par les migrants-retour; ce dernier facteur - le coût, en temps, d'accès à l'eau potable - renvoyant à un état de fait datant de bien avant les récentes années de sécheresse, il n'est pas étonnant qu'il soit d'avantage cité par le groupe de migrants installés durablement en ville et dont la migration, dans une bonne proportion de cas antérieure à la grande sécheresse, est davantage liée à des causes structurelles, moins "contrainte» par l'irruption de la catastrophe «naturelle », et relève davantage de stratégies à long terme. Les causes directes alléguées par les acteurs différencient là encore assez bien les deux groupes de migrants et renvoient tant à leur insertion économique qu'à leur mode de vie ( $\mathrm{Khi}^{2}$ : diff. sign. ; seuil : $\left.5 \%\right)$.

La migration, qu'elle relève d'une "contrainte» survenue brutalement ou d'une décision stratégique plus longtemps mûrie, n'implique pas seulement le départ du village mais suppose aussi de savoir où aller. Différents facteurs pouvaient "attirer " les résidants d'al-Halba vers al-Dueim, et les deux groupes de migrants pouvaient ainsi se différencier selon le poids que prenaient ces facteurs dans leur décision d'y immigrer. Ces derniers se présentaient dans le questionnaire selon les modalités suivantes : opportunité d'emploi (connue avant le départ) dans cette ville, ou présence de parents et/ou de relations, ou familiarité avec cette ville (séjours antérieurs, etc.), plus une rubrique "autre». En lait, les deux groupes ne se différencient pas significativement selon ces modalités: la présence de parents et/ou de relations domine nettement dans les deux cas, suivie de la familiarité antérieurement acquise avec la ville et de la connaissance des possibilités d'emploi y existant. Tout au plus retrouve-t-on en filigrane l'opposition entre migrants-retour, plus «traditionnels » et comptant davantage sur des relations familiales (50\% contre $33 \%$ pour les autres migrants) et ceux qui sont restés à al-Dueim, qui affirment une plus grande familiarité antérieure avec cette ville ( $38 \%$ contre $21 \%$ de migrants-retour se trouvant dans ce cas) et qui ont davantage décidé d'y migrer.

Enfin, dans la mesure où, comme l'écrit Shami (op. cit.), «les mouvements de population, volontaires ou involontaires, individuels ou en groupes, sont en interrelation et s'engendrent les uns les autres ", il était intéressant, pour reprendre la problématique évoquée en introduction, de tester, avant et après leur départ vers la ville, le degré d'intégration de chacun des deux groupes de migrants au marché du travail saisonnier de la cueillette du coton (en Guezira, essentiellement). Avant migration, $82 \%$ des familles retournées ultérieurement au village avaient au moins un membre pratiquant ce type de travail, contre $53 \%$ des familles de migrants restées à al- 
Dueim. Après migration, donc une fois installées provisoirement ou définitivement en ville, $86 \%$ des familles se trouvant dans le premier cas pratiquaient ce type de travail (donc une proportion encore plus élevée que lorsqu'elles résidaient au village), alors que $19 \%$ seulement des familles se trouvant dans le second continuaient à se déplacer pour effectuer la récolte du coton (soit une baisse des trois cinquièmes par rapport à la situation avant émigration) ( $\mathrm{Khi}^{2}$ : diff. sign.; seuil : $1 \%$ ). Il semble donc que le projet d'installation définitive en ville s'accompagne d'un véritable changement du mode de vie et de sources de revenus, donc de travail, alors que les familles retournées ultérieurement au village n'ont jamais abandonné cette composante essentielle à la fois de leur revenus et de leur cycle annuel qu'est la migration saisonnière vers les périmètres cotonniers.

41 Deux modes de vie s'expriment donc à travers les variables retenues et correspondent, de manière non mécanique, aux deux statuts migratoires qui distinguent les sousgroupes enquêtes. Alors qu'il n'est pas étonnant que ces deux modes de vie opposent après migration ou ré émigration - ces deux sous-groupes, il est beaucoup plus intéressant de voir apparaître des oppositions les différenciant avant migration, la moindre n'étant pas celle qui distingue un groupe de migrants " sous contrainte ", plus agricoles, dont l'émigration en ville n'est qu'une étape avant le retour au village, d'un groupe de migrants moins liés à l'agriculture et plus alphabétisés qui, pour une part d'entre eux, ont décidé de migrer en ville - et ceci avant même la sécheresse - et d'y organiser leur vie en utilisant les ressources qui s'y trouvent (éducation, diversification des emplois et des biens de consommation).

\section{BIBLIOGRAPHIE}

Adra, Report of the second Nutrition Survey in Northern While Nile Province, Central Region, June/July 1986, 1987.

Bardhan P., Land, Labour and Rural Poverty: Essays in Development Economics, Columbia University Press, New York. 1984.

Booth A., « The Development of the Swazi Labour Market 1900-1968 ", South African Labour Bulletin : Focus on Swaziland, vol. 7, n 6,1982.

De Jong G. F., « Population Redistribution Policies: Alternatives from the Netherlands, Great Britain and Israel », Social Science Quarterly, September, 1975.

ILO/JASPA Mission, Employment and Economic Reform: Towards a Strategy for the Sudan, ILO Office, Geneva, 1988.

Jaycox E. V. K., « Ending Hunger in Africa », African Report, September-October, 1988, pp. 15-18.

Kakwani N., « Testing for significance of Poverty Differences », LMMS Staff Working Papers $\mathrm{n}^{\circ}$ 597,1990 .

Mohammed A. T., Rural-Rural Labour Circulation and Poverty: The Evidence From the Sudan Seasonal Migration. Unpublished Ph.D. Thesis. University of Pennsylvania States. 1989. 
O'Brien J., Agricultural Labour and Economic Development in the Sudan, Unpublished Ph.D. Thesis, University of Connecticut, 1979.

Polachek S. W. and Horvath F. W., « A Life Cycle Approach to Migration: Analysis of the Perspicacious Peregrinator », in R. Ehrenbery (ed.), Research in Labour Economies, vol. 1, 1977.

Prothero, Mansell \& Coppman, Circulation in Third World Countries, 1985.

Shami S., «The Social Implications of Population Displacement and Resettlement: an Overview with Focus on the Middle East », Population Council, West Asia and North Africa. Regional Papers, June, $\mathrm{n}^{0} 37.1990$.

United Nations, Population Distribution. Migration and Development. U.N., New York, 1984.

Willekens F., « Migration and Development: A Micro Prospective », Paper presented at the IUSS Seminar on Internal Migration and Regional Development, Montreal, April 1-3, Working Paper $\mathrm{n}^{\circ}$ 2, August 1985.

INDEX

Mots-clés : Soudan

\section{AUTEUR}

AHMED TAHA MOHAMMED

Université de Khartoum 